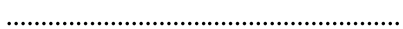

AN. MED. INTERNA (Madrid) Vol. 19, N. $^{\circ} 5$, pp. $246-250,2002$

\title{
Fístula aortoentérica secundaria a infección de prótesis aortobifemoral
}

\author{
F. GABRIEL BOTELLA, M. LABIÓS GÓMEZ, L. IBÁÑEZ GADEA, L. FÁCILA \\ RUBIO, C. CARBONELL CANTÍ*
}

\section{Servicio de Medicina Interna. *Servicio de Cirugía Cardiovascular. Hospital Clínico} Universitario de Valencia

\author{
AORTOENTERIC FISTULA SECONDARY TO PROSTHESIS AORTO - \\ BIFEMORAL INFECTION
}

\section{RESUMEN}

Presentamos el caso de un hombre de 76 años, intervenido de una obstrucción iliaca bilateral mediante colocación de una prótesis aortobifemoral, que cinco años después presentó dolor en la fosa iliaca izquierda y fiebre en agujas de $39^{\circ} \mathrm{C}$. En la exploración física destacaba un abdomen doloroso en la fosa iliaca izquierda con signos de irritación peritoneal. En las pruebas de laboratorio se detectó una leucocitosis con neutrofilia y hemocultivos negativos. La tomografía computadorizada (TC) objetivó la presencia de burbujas de gas alrededor de la prótesis, así como una colección líquida con áreas necróticas en su interior que afectaba a los músculos psoas e iliaco. En la misma exploración, la punción aspirativa con drenaje del absceso demostró en los cultivos realizados en medios aerobios la presencia de Enterococcus faecalis y Enterobacter cloacae. Al presentar bruscamente una hemorragia gastrointestinal alta, se le practicó una endoscopia gastroduodenal en la que se evidenció una fístula aortoduodenal con sangrado activo. Cuando se le iba a practicar un bypass extraanatómico, el enfermo falleció al presentar un shock hipovolémico brusco, que no respondió al tratamiento pertinente.

Analizamos los criterios diagnósticos actuales de infección de las prótesis vasculares y su complicación más grave, la fistula aortoentérica (FAE), que aparece en el 0,3-5,9\% de los pacientes que sufren reconstrucciones protésicas de la aorta abdominal, ya sea por enfermedades oclusivas o aneurismáticas. Destacamos la importancia de realizar un diagnóstico precoz de la infección de la porción retroperitoneal del injerto vascular que, a menudo, se manifiesta con signos clínicos sutiles y no específicos, con las técnicas actualmente disponibles como: la TC, la punción aspirativa guiada por ella, y la angiografía. Todo esto, con el fin de erradicar el proceso infeccioso y disminuir las tasas de mortalidad, desde las actuales del $43 \%$, hasta las más optimistas estimadas en un $19 \%$.

PALABRAS CLAVE: Infección de injertos vasculares. Fístula aortoentérica. Tomografía computadorizada. Angiografía.

\begin{abstract}
We present the case of a 76 year-old man, intervened of an obstruc tion bilateral iliac by means of placement of a prosthesis aortobifemoral that presented pain in the grave left iliac and fever in needles of $39^{\circ} \mathrm{C}$ to the five years of the intervention. In the physical exploration it highligh ted a painful abdomen in the grave left iliac with signs of peritoneal irri tation. In the laboratory tests a leukocytosis was detected with neutrophi lia and negative culture. The computed thomography (CT) show the presence of gas bubbles around the prosthesis, as well as a liquid collection with areas necrotics in their interior that affected to the psoas and iliac muscles. In the same exploration the aspirative puncture with drai nage of the absces demonstrated in the cultivations carried out in aero bic means the presence of Enterococcus faecalis and Enterobacter cloa cae. When presenting a high gastrointestinal hemorrhage abruptly, he was practiced and gastroduodenal endoscope in which a aortoduodenal fistula was evidenced with having bled active. When a bypass extra-ana tomic, the sick person will practice it died when presenting a shock abrupt hipovolemic that he didn't respond to the pertinent treatment. We analyze the approaches current diagnoses of infection of the vascular prosthesis and their more serious complication, the aortoenteric fistula $(A E F)$ that either appears in the 0,3-5,9\% of the patients who undergo prosthetic reconstruction of the abdominal aorta, for oclusive or aneu rismal disease. We highlight the importance of carrying out a precocious diagnosis of the infection of the portion retroperitoneal of the vascular graft that, often, it is manifested with subtle and not specific clinical signs, with the techniques at the moment available as: the CT, fine need le aspiration guided by her, and to diminish the rates of mortality, from the current of $43 \%$, until the most optimistic estimated in $19 \%$.
\end{abstract}

KEY WORDS: Vascular graft infection. Aortoenteric fistula. Computed tomography. Angiography.

Gabriel Botella F, Labiós Gómez M, Ibáñez Gadea L, Fácila Rubio L, Carbonell Cantí C. Fístula aortoentérica secundaria a infección de pró tesis aortobifemoral. An Med Interna (Madrid) 2002; 19: 246-250.

\section{INTRODUCCIÓN}

Las infecciones de las prótesis aortoilíacas constituyen una de las complicaciones más temidas y catastróficas de la cirugía vascular reparadora. Afortunadamente, su incidencia es baja ya que oscila entre el 1 y el $6 \%$ de los injertos implantados con una media del 2,5\%en la mayor parte de los estudios realizados $(1,2)$. Su gravedad no sólo estriba en su elevada mortalidad,que puede variar entre el 25 y el $75 \%$ de los casos para los injertos suprainguinales (3) y alrededor del $22 \%$ para los infraingui-

Trabajo aceptado: 31 de octubre de 2000

Correspondencia: Francisco Gabriel Botella. C/ Conde Altea 49. 46005 Valencia. 
nales $(4,5)-$,sino también en la alta tasa de amputaciones, que para estos últimos puede llegar al 79\% (5). En la mitad de los casos, por lo menos, las manifestaciones infecciosas aparecen durante los primeros cuatro meses después de la operación,infección precoz $(2,4)$-, mientras que en la otra mitad puede ocurrir hasta diez años después de la misma,- infección tardía (6)-. Es en este último período donde las infecciones larvadas de las prótesis vasculares, sin manifestaciones clínicas ni biológicas de ningún tipo, erosionan la sutura aórtica y la pared duodenal o intestinal, desarrollando una fístula aortoentérica que aparece en el 0,3-5,9\% de los pacientes que sufren reconstrucciones protésicas de la aorta abdominal, ya sea por enfermedades oclusivas o aneurismáticas $(7,8)$. Una hemorragia gastrointestinal alta e intermitente, manifestada como una hematemesis y/o melena, puede ser el único hallazgo clínico. En estos casos un alto índice de sospecha de esta complicación, después de excluir un sangrado activo de una úlcera gastroduodenal por gastroscopia, hace necesaria una exploración quirúrgica urgente antes de que una hemorragia masiva acabe definitivamente con la vida del paciente (9). Exploración quirúrgica que en la mayor parte de los casos sirve para la realización de un bypass extraanatómico seguido inmediatamente de la extracción de la prótesis infectada, aunque cada vez se utilizan con mayor profusión los homoinjertos arteriales y las prótesis impregnadas de antibióticos $(2,10)$.

Presentamos un caso de fístula aortoentérica (FAE) secundaria a la infección tardía de una prótesis aortobifemoral en un paciente de 76 años, intervenido, hacía cinco, de una obstrucción iliaca bilateral. Destacamos la importancia capital de realizar un diagnóstico precoz de la infección de la porción retroperitoneal del injerto aórtico que, a menudo, se manifiesta con signos y síntomas clínicos sutiles y no específicos, cuando no ausentes, lo que hace extremadamente dificultoso el diagnóstico. Hacemos especial hincapié en el uso juicioso pero decidido, de las técnicas diagnósticas actualmente disponibles como: la tomografía computadorizada (TC) con contraste para comprobar la existencia de abcesos, de las interfases airelíquido, de las burbujas de gas, o de los falsos aneurismas (10); la angiografía, para delimitar la anatomía vascular (11); y la punción aspirativa guiada con TC, para determinar el germen que lo provoca y sus estudios de sensibilidad correspondientes (10). Todo ello con el fin de erradicar el proceso infeccioso por una parte y disminuir las tasas de mortalidad por otra, desde las actuales del 43\%, hasta las más optimistas estimadas en un $19 \%$.

\section{CASO APORTADO}

Varón de 76 años, intervenido por obstrucción ilíaca bilateral mediante colocación de una prótesis aortobifemoral bilateral cinco años antes. Ingresado en nuestro servicio por presentar desde hacía cuatro meses dolor en fosa iliaca izquierda y fiebre en agujas de hasta $39^{\circ} \mathrm{C}$. En la exploración física destacaba una temperatura axilar de $38,7^{\circ} \mathrm{C}$, un abdomen doloroso en la fosa ilíaca izquierda, con signos de irritación peritoneal y peristaltismo conservado. Tacto rectal doloroso, con heces normales. Los hallazgos de laboratorio incluyen unos leucocitos de 16.580, Hematies: 3.460.000, Hemoglobina: 9,2 g/dL, Hematocrito: 28\%, VCM: 87 fL, Plaquetas: 483.000/mm3 , Neutrófilos: 90\%, VSG: $106 \mathrm{~mm}$, LDH: $1027 \mathrm{UI} / \mathrm{L}$. Fué tratado inicialmente con cefotaxima y metronidazol, previos hemocultivos seriados -9-, todos ellos negativos. El ecodoppler abdominal demostró una colección líquida en fosa ilíaca izquierda y la TC abdominal realizada con contraste, la existencia de una marcada dilatación aórtica infrarrenal (Fig. 1), con pequeñas burbujas de gas distribuidas alrededor de la prótesis, así como una colección líquida con áreas necróticas en su interior que afectaba a los músculos psoas e ilíaco. En la misma exploración se practicó una punción aspirativa del absceso con drenaje permanente del mismo, del que se extrajeron $100 \mathrm{~cm} 3$ de un líquido purulento. Los cultivos realizados, en medios anaerobios y aerobios, demostraron en estos últimos la presencia de Enterococcus faecalis y Enterobacter cloacae, sensibles ambos al carbapenem, por lo que se procedió al cambio de antibioterapia desapareciendo la fiebre en cuarentaiocho horas. Realizada aortografía y arteriografía de los miembros inferiores para planificar el tipo de reconstrucción vascular, demostró la ausencia de sangrado gastrointestinal y la permeabilidad del bypass aortofemoral derecho, con la obstrucción completa del izquierdo (Fig. 2). En el miembro inferior derecho se apreció una obstrucción completa de la arteria femoral superficial, visualizándose la arteria femoral profunda con repermeabilización en la arteria poplítea. En el miembro inferior izquierdo se evidenció la arteria femoral profunda con la arteria poplítea permeable. Durante su hospitalización, el paciente presentó bruscamente una hemorragia gastrointestinal alta que requirió la práctica de una endoscopia gastroduodenal, en la que se evidenció una fístula aortoentérica con sangrado activo (Fig. 3). Cuando se le iba a practicar un bypass extraanatómico y extracción posterior de la prótesis infectada, el enfermo falleció al presentar un schock hipovolémico brusco, que no respondió al tratamiento pertinente.

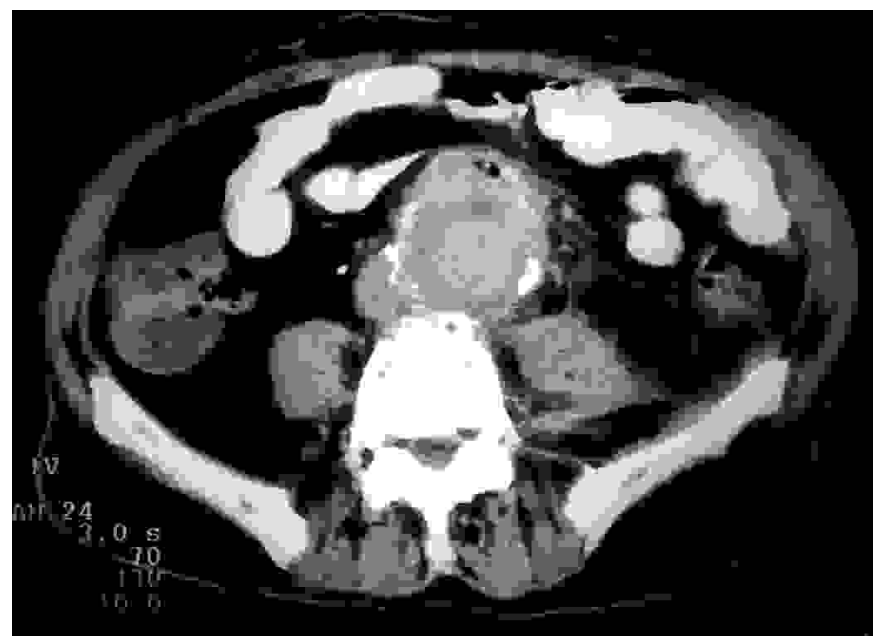

Fig. 1. TC abdominal: dilatación aórtica infrarrenal, con burbujas de gas alrededor de la prótesis aortoilíaca.

\section{DISCUSIÓN}

En los últimos años, la cirugía vascular se ha beneficiado de la introducción y desarrollo de los nuevos biomateriales utilizados en los injertos para el tratamiento de las enfermedades vasculares periféricas $(11,12)$. Las complicaciones asociadas a este tipo de prótesis incluyen las trombosis, los embolismos, los hematomas y la rotura del vaso, además de las infecciones. Aunque estas últimas son infrecuentes, sin embargo, presentan una elevada morbimortalidad (11).

También en los últimos años ha habido un cambio gradual en el modelo de infección. En los primeros estudios, el Staphy lococcus aureus era el responsable del $50 \%$ de todas las infecciones de los injertos vasculares. Más recientemente su incidencia ha disminuido y actualmente ocupa el primer lugar el Staphylococcus epidermidis con el $60 \%$ de todas ellas (4). 


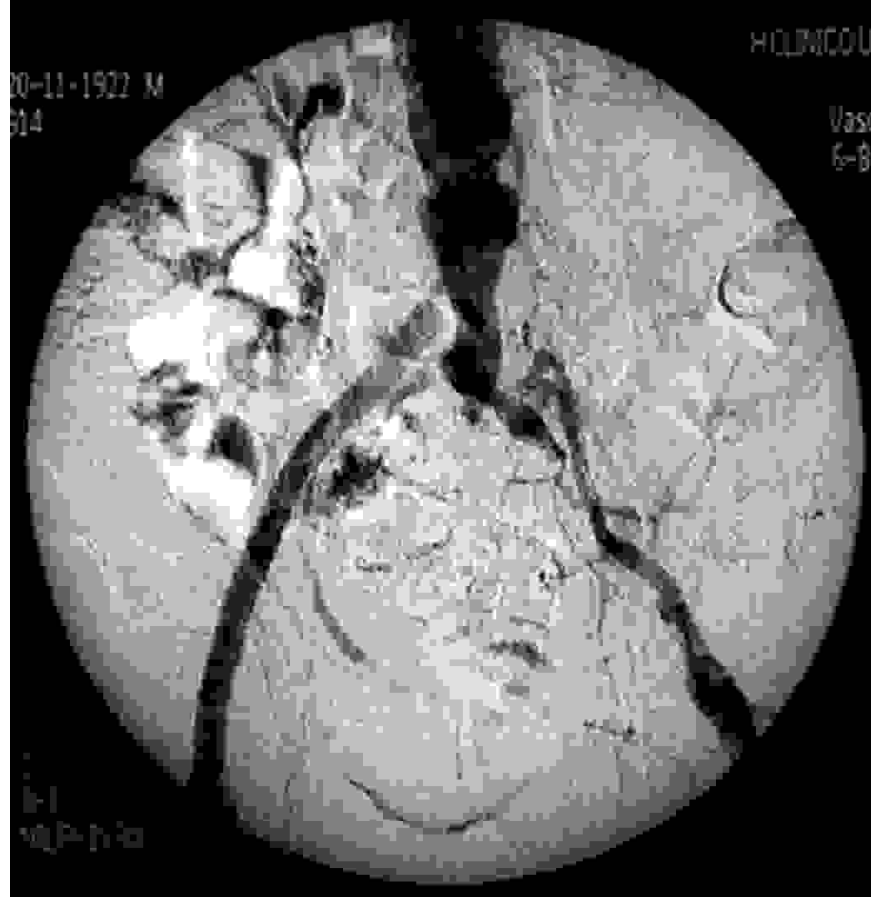

Fig. 2. Aortografía en la que se aprecia la permeabilidad del bypass aortofemoral derecho, con obstrucción completa del izquierdo.

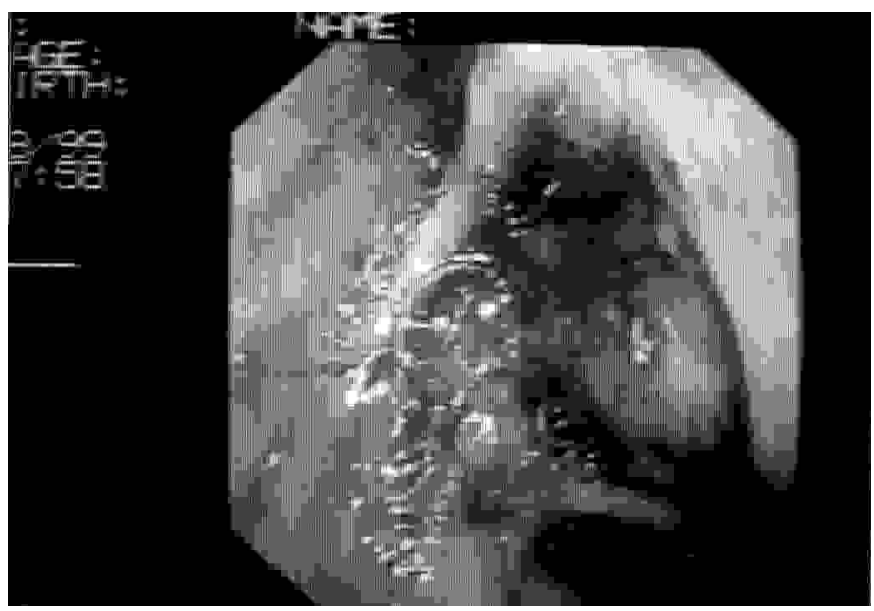

Fig. 3. Endoscopia gastroduodenal: fístula aortoentérica en la tercera porción duodenal. La prótesis aortoilíaca se observa al fondo.

Diferentes estudios han demostrado que el período de latencia entre la implantación de las prótesis y la aparición de los signos de infección de la mismas se concentran alrededor de dos fechas: a los cuatro meses $(2,4,13)$, y a los seis años $(2,6)$. Esto ha permitido dividir las infecciones en dos grupos: precoces $(2,4)$, cuando los signos de infección aparecen antes del primer año, y tardías $(2,4,6)$, cuando ha transcurrido más de un año desde su implantación, como en nuestro caso. En las primeras, los patógenos más frecuentemente identificados incluyen S.aureus coagulasa positivos y las enterobacterias, principalmente Escherichia coli y Proteus, aunque los enterococos y la Pseudomona aeruginosa también son aislados. En las segundas, el más comúnmente identificado es el S. epidermidis coagulasa negativo, y, en menor proporción, los hongos $(4,14)$.
Dos características principales contribuyen a la severidad de ambos tipos de infección: en primer lugar, la ausencia casi completa de signos clínicos específicos, particularmente en la fase precoz, lo que hace posible el diagnóstico únicamente cuando el pus está rodeando parcial o totalmente a la prótesis, o se haya producido una FAE $(6,13)$, extremos ambos que se dieron en nuestro paciente. En segundo lugar, por su extremada resistencia a la quimioterapia debido a los fenómenos de escape utilizados por los distintos agentes microbianos para su propia supervivencia , al desarrollar complejas estrategias de evasión a la respuesta antiinfecciosa del huésped $(6,15)$. Es ampliamente aceptado que la capacidad bacteriana para adherirse y sobrevivir en superficies inertes es la consecuencia de la producción de un polisacárido polimérico extracelular que contiene substancias conocidas como glycocalyx (mucina) que se une a la prótesis y tiene una acción protectora al reducir la penetración de los antibióticos e inhibir la acción de los mismos $(4,13,16)$. El organismo induce una respuesta inflamatoria de bajo grado durante un largo período de tiempo que desemboca en la formación de un aneurisma anastomótico o en una FAE, que puede provocar la muerte del paciente $(5,7,9)$, como ocurrió en nuestro enfermo. Este mecanismo de adhesión pasa por una fase inicial proteíca y una posterior esopolimérica (13). Se cree que esta última es la que permite a las bacterias resistir fuertemente a las adversas condiciones y sobrevivir a los agentes antibacterianos y a las defensas del huésped in vivo $(17,18)$. Este peculiar mecanismo de desarrollo, que implica la adhesión a las superficies inertes y el crecimiento en microcolonias, progresivamente amplía la anchura del biofilm (interfase mucina-biomaterial). En él, el status fisiológico de las distintas células bacterianas que lo habitan es heterogéneo, lo que viene determinado por la localización de cada una de ellas dentro de las distintas capas celulares que componen el biofilm. Esto les hace tener un comportamiento semejante al de los tejidos animales $(13,18)$. Las células localizadas en las capas más externas tienen libre acceso a los nutrientes y al oxígeno y pocos problemas en la eliminación de sus productos de desecho. Pero también son susceptibles a los agentes antibacterianos y a los elementos celulares fagocíticos encargados de su destrucción, representados por los polimorfonucleares y los macrófagos $(13,18)$. Además, la activación de la vía alternativa del sistema de complemento por el peptidoglicano de las bacterias gram positivas y el lipopolisacárido de las gram negativas también desempeña una función relevante (15). Por el contrario, las situadas en las capas más profundas del biofilm tienen un escaso acceso al oxígeno y a los nutrientes, y grandes problemas para eliminar sus productos de desecho. Todo ello les hace tener una mínima, cuando no nula, actividad reproductora y un metabolismo especializado en la síntesis de esopolímeros. Consecuentemente, son extremadamente resistentes a los antibacterianos, al permanecer casi siempre en un estado de latencia (13). Por otra parte, dadas las funciones del glycocalyx como matriz de intercambio iónico, capaz de atrapar distintos componentes, las bacterias del biofilm dedican parte de su actividad a la producción de enzimas implicados en la resistencia activa contra los agentes antibacterianos, subsiguientemente externalizados y concentrados en la matriz esopolimérica $(19,20)$. Estas diferencias celulares son proporcionales a la edad del biofilm, así como su resistencia a los agentes antibacterianos (18). La consecuencia de todo ello a nivel clínico es que en muchas ocasiones los hemocultivos realizados son repetidamente negati- 
vos $(2,4)$, al manifestarse la infección por cortos, pero dramáticos períodos de fiebre en los que sólo las bacterias de las capas celulares más externas pasan al torrente sanguíneo. Esto obliga a efectuar hemocultivos seriadamente, durante breves períodos de tiempo en los que incluso pueden ser neutralizadas por las defensas del huésped, fundamentalmente por la producción de IgG inducida por células Th2, principal mecanismo molecular de defensa contra las bacterias extracelulares (15). Además no hay que olvidar que muchos de estos enfermos, previamente, han sido sometidos empíricamente a antibioterapias prolongadas antes de llegar al hospital, lo que contribuye a que los hemocultivos sean negativos $(2,4)$.

Establecer por tanto el diagnóstico de certeza de la infección de una prótesis aórtica es sin duda la primera dificultad que debemos salvar. La amplia disponibilidad actual de la TC, la convierte en la primera exploración a realizar en todo paciente con sospecha de padecerla (6). Los criterios diagnósticos incluyen (10): la presencia de colecciones líquidas periinjerto,- puestas de manifiesto hasta en el $100 \%$ de los casos (2) -, de burbujas de gas y la alteración de la densidad de los tejidos blandos (músculos psoas e iliaco). Los leucocitos marcados con radioisótopos se reservan para aquellos casos en los que los resultados de la TC son contradictorios. La aspiración de la colección líquida con aguja fina guiada con TC tiene una alta sensibilidad para confirmar la infección, como ocurrió en nuestro caso. La aortografía sirve para excluir otras fuentes de sangrado gastrointestinal, definir la anatomia vascular y planificar la reconstrucción vascular $(7,11)$. Ninguna prueba es lo consistentemente exacta como para diagnosticar una FAE, aunque la presencia de aire alrededor de la prótesis y fuera de la luz intestinal es altamente sugestiva de ella (7). La endoscopia gastroduodenal puede confirmar el diagnóstico si la prótesis es observada a través del endoscopio, como ocurrió en nuestro caso. Las series gastrointestinales superiores son también diagnósticas si el contraste es identificado fuera de luz intestinal y alrededor del injerto vascular. Sin embargo, un tercio de los pacientes requerirán la práctica de una laparotomía para establecer el diagnóstico definitivo (7).

El éxito del tratamiento quirúrgico siempre es problemático al depender tanto de la intensidad de la hemorragia como del grado de infección. Aquellos pacientes que presentan una hemorragia cataclísmica, como en nuestro enfermo, no tienen ninguna posibilidad de supervivencia sin laparotomía $(2,7,10)$. El tratamiento clásico de la FAE es la revascularización extraanatómica de las extremidades inferiores seguida por una laparotomía en la que se cierra la fístula duodenal y el muñón aórtico infrarrenal, resecándose por último el injerto infectado $(2,7,10)$. El principal inconveniente de esta técnica es su elevada mortalidad precoz $(19 \%)$, y la alta tasa de reinfecciones $(26 \%)$ lo que obliga a someter a los pacientes a numerosas reintervenciones con el paso del tiempo (21-23). Para evitar todos estos inconvenientes se están realizando actualmente estudios prospectivos sobre la reconstrucción in situ con las nuevas prótesis de politetrafluoroetileno (PTFE), que presentan una adherencia bacteriana diez a cien veces menor que las antiguas de dacron, los homoinjertos arteriales o los injertos autógenos de venas femorales y safenas $(2,10,12)$. Para extraer conclusiones sobre la bondad de los primeros es necesario esperar un mínimo de cinco años (12). Para los segundos, la mortalidad precoz oscila entre el 22$30 \%$, según las series estudiadas $(24,25)$, lo que parece lógico dado que el principal inconveniente de estos injertos es la dilatación producida por una disminución de la elasticidad de la lámina media, lo que obligará a substituirlos en una proporción todavía por determinar (2). Después de la intervención, a los pacientes se les debe de administrar una antibioterapia sistémica durante dos semanas, generalmente una cefalosporina y metronidazol, o hasta que los estudios de sensibilidad estén disponibles. Posteriormente, se aconseja proseguir con una antibioterapia oral apropiada durante seis semanas o hasta que desaparezcan los signos clínicos de infección (10).

A pesar de que el pronóstico de los pacientes con FAE ha mejorado en los últimos años, la mortalidad, la pérdida de los miembros inferiores y las complicaciones infecciosas son, todavía, elevadas. Aquellos que presentan hemorragias gastrointestinales, con signos clínicos de infección, tienen una alta mortalidad, a pesar de las nuevas técnicas quirúrgicas introducidas en los últimos años. Finalmente la reconstrucción in situ, actualmente en fase experimental, parece provocar menos infecciones tardías, aunque hasta ahora solo

se ha aplicado en pacientes seleccionados y no existe protocolo que justifique la posible aplicación, con el mismo grado de efectividad a la generalidad de los enfermos.

\section{Bibliografía}

1. O'obrien T, Collin T. Prosthetic vascular graft infection. Br J Surg 1992; 79: 1262-7.

2. Rodríguez A, Royo J, Bofill R, et al. Tratamiento quirúrgico de las infecciones de las prótesis arteriales del sector aortoilíaco: una década de experiencia. Angiología 1996; 2: 61-8.

3. Calligaro KD, Veith FJ. Graft preserving methods for managing aortofemoral prosthetic graft infection. Eur J Vasc Endovasc Surg 1997; 14 (supplement A): 38-42.

4. Hicks RC, Greenhalgh RM. The pathogenesis of vascular graft infection. Eur J Vasc Endovasc Surg 1997; 14 (supplement A): 5-9.

5. Kikta MJ, Goodman LJ, Bishara RA. Mortality and limb loss with infected infrainguinal bypass graft. J Vasc Surg 1987; 5: 566-71.

6. Spartera C, Morettini G, Bafile G, Di Cesare E, Alagia G, Ventura M. Diagnostic imaging techniques in vascular graft infection. Eur $\mathrm{J}$ Vasc Endovasc Surg 1997;14 (supplement A): 24-6.
7. Menawat SS, Gloviczki P, Seny RD, Cheny KJ, Bower TC, Hallett JW. Management of aortic graft-enteric fistulae. Eur J Vasc Endovasc Surg 1997; 14 (supplement A): 74-81

8. van den Broek N Th, Eikelboom BC. Aortic anastomosis surveillance. Eur J Vasc Endovasc Surg 1997; 14 ( supplement A): 30-4.

9. Balas P. An overview of aortofemoral graft infection. Eur J Vasc Endovasc Surg 1997; 14 (supplement A): 3-4

10. Naylor AR. Aortic prosthetic infection. Br J Surg 1999; 86: 435-6.

11. Sheeran SR, Gestring ML, Murphy TP, Slaiby JM. Endovascular graft-related iliac artery infection. J Vasc Interv Radiol 1999; 10: 877 882.

12. Grupo Español de investigación P.A.S.G. (Prótesis Aórtica <strecht>Goretex). Evaluación de una nueva prótesis de PTFE (strecht) en el sector aórtico. Análisis prospectivos en 221 pacientes. Angiología 1999; 5: 209-18 
13. Selan L, Passariello C. Microbiological diagnosis of aortofemoral graft infections. Eur J Vasc Endovasc Surg 1997; (supplement A):10-12.

14. García R, Clará A, di Stefano S, Legarra J, Sarralde JA. An unusual vascular graft infection by Aspergillus. Angiology 1999; 50: 169-73.

15. Manzano L, Rodriguez M, Alvarez-Mon M. Respuesta inmunitaria frente a microorganismos. Med Clin (Barc) 1999; 113: 505-11.

16. Henke PK, Bergamini TM, Garrison JR, Brittian KR, Peyton JC, Lam TM. Staphylococcus epidermidis graft infection is associated with locally suppressed major histocompatibility complex class II and elevated MAC-1 expression. Arch Surg 1997; 132: 894-902.

17. Giwercman B, Jensen ET, Hoiby N, Kharazmi A, Costerton JW. Induction of b-lactamase production in Pseudomonas aeruginosa biofilm. Antimicrob Agents Chemother 1991; 35: 1008-10.

18. Anwar H, Strap JL, Costerton JW. Establishment of aging biofilms: possible mechanism of bacterial resistance to antimicrobial therapy. Antimicrob Agents Chemother 1992; 36: 1347-51.

19. Costerton JW, Irvin RT. The bacterial glycocalyx in nature and disease. Ann Rev Microbiol 1981; 35: 299-324
20. Costerton JW, Cheng KJ, Geesey CG, et al. Bacterial biofilms in nature and disease. Ann Rev Microbiol 1987; 41: 435-64.

21. Reilly LM, Stoney RJ, Goldstone J, Ehrenfeld WK. Improved management of aortic graft infection: The influence of operation sequence and staging. J Vasc Surg 1987; 5: 421-31.

22. Kuestner LM, Reilly LM, Jicha DL, Ehrenfeld WK, Goldstone J, Stoney RJ. Secondary aortoenteric fistula: contemporary outcome with use of extra-anatomic bypass and infected graft excision. J Vasc Surg 1995: 29 McCann RL, Schwartz LB, Georgiade GS. Management of abdominal aortic graft complications. Ann Surg 1993; 217: 729-34.

23. Henke P, Bergamini M, Rose S, Richardson J. Current options in prosthetic vascular graft infection. Am Surg 1998; 64:3 9-45.

24. Walker WE, Cooley DA, Duncan JM, Hallman GL, OTT DA, Reul GJ. The management of aortoduodenal fistula by in situ replacement of the infected abdominal aortic graft. Ann Surg 1987; 205: 727-32.

25. Jacobs MJ, Reul GJ, Gregoric I, Cooley DA. In-situ replacement and extra-anatomic bypass for the treatment of infected abdominal aortic grafts. Eur J Vasc Surg 1991; 5: 83-86. 